\title{
A Mobile and Desktop Application for Enhancing Group Awareness in Knowledge Work Teams
}

\author{
Timo Saari ${ }^{1}$, Kari Kallinen ${ }^{2}$, Mikko Salminen ${ }^{2}$, Niklas Ravaja ${ }^{2}$, and Marco Rapino ${ }^{2}$ \\ ${ }^{1}$ Temple University, 1801 N. Broad Street, Philadelphia, PA, USA, and Center for Knowledge \\ and Innovation Research (CKIR), Helsinki School of Economics, Finland, and Helsinki \\ Institute for Information Technology (HIIT), Finland \\ saariatemple. edu \\ ${ }^{2}$ Center for Knowledge and Innovation Research (CKIR), Helsinki School of Economics, \\ Finland \\ Kari.Kallinen@hse.fi, Mikko.Salminen@hse.fi, \\ Niklas.Ravaja@hse.fi, Marko.Rapino@hse.fi
}

\begin{abstract}
In this paper we present a first prototype for a mobile and desktop system and application for enhancing group awareness in knowledge work teams. The prototype gathers information from the interactions of the group within the application and analyses it. Results are displayed to members of the group as key indexes describing the activity of the group as a whole and the individual members of the group. The advantages of using the prototype are expected to be increased awareness within group possibly leading to positive effects on group performance.
\end{abstract}

Keywords: Group awareness, emotional awareness, knowledge work, mobile application, desktop application.

\section{Introduction}

We see knowledge work consisting of the capacity to act in intelligent ways in one's context and environment. Senge [1] suggests that while information implies knowing "about" things, and is received and passed on, knowledge implies knowing "how", thereby giving people the capacity for effective action. Davenport et al. [2] define knowledge work as "the acquisition, creation, packaging, or application of knowledge. Characterized by variety and exception rather than routine, it is performed by professional or technical workers with a high level of skill and expertise."

Consequently, knowledge work includes the creation of knowledge, the application of knowledge, the transmission of knowledge, and the acquisition of knowledge.

McGrath and Hollingshead [3] have proposed that technologies, as they have been applied to groups, can be placed along a dimension of increasing and decreasing richness of social cues. Face-to-face groups have access to a rich variety of social cues that they can then use to determine the preferences and positions of other group members. On the other hand, computer-mediated groups, do not have access to nonverbal cues, and must rely simply on the written word. That is, computer mediated groups and hence computer mediated group work are low in the richness of social cues. 
There are different types of group awareness some of which are relevant to worklike tasks. According to Greenberg [4], there are several types of group awareness needed for effective collaboration:

- Workspace awareness is "the up-to-the minute knowledge a person requires about another group member's interaction with a shared workspace if they are to collaborate effectively".

- 'Group-structural awareness involves knowledge about such things as people's roles and responsibilities, their positions on an issue, their status, and group processes."

- "Informal awareness of a work community is basic knowledge about who is around in general (but perhaps out of site), who is physically in a room with you, and where people are located relative to you."

- "Social awareness is the information that a person maintains about others in a social or conversational context: things like whether another person is paying attention, their emotional state, or their level of interest." Other information can be the special skills a co-worker has.

Also, emotional awareness within a group has been discussed in relation to knowledge work groups [see 5]. Within this article we see emotional awareness, i.e. awareness of emotion and mood states of the members of the group as part of social awareness.

Often in knowledge work situations, awareness of others provides information that is essential for frictionless and effective collaboration. Even though group awareness is taken for granted in face-to-face work, it is rather difficult to maintain in distributed settings. Hence, there is a considerable challenge in designing technology to support the types of group awareness that actually may lead to increased performance or other beneficial effects at work.

We propose an application to increase awareness in knowledge work groups. Our application enhances group awareness by making explicit the implicit interaction patterns in a group. We expect our application to have beneficial effects on performance in knowledge processes and tasks.

\section{Use Scenario and System Design}

\section{Supporting general knowledge work processes}

Knowledge work tasks can roughly be classified to job specific tasks and general processes [see 6]. Job-specific tasks differ greatly as a function of the type of work. Examples are preparing a budget, analyzing results in terms of estimated and actual costs, planning and scheduling a project, eliciting and documenting system requirements, and writing applications software. There are also many general processes when working. General processes can be such as goal setting, communication, updates, group cohesion and synchrony maintenance and informal group communication and coordination.

"Mobile" knowledge work differs mostly from "normal" knowledge work in that it takes place in distributed settings with the use of mobile technologies. Mobile knowledge work can be mostly mobile such as when a person is communicating with others 
and accessing files on the field while conducting work tasks. Mobile knowledge work is naturally intertwined with non-mobile knowledge work as people move in and out of their offices. Mobile technologies for knowledge work also mix with desktop computing environments as workers also carry their mobile phones to the office.

Our use scenario is enhancing general communication processes of knowledge work teams. We feel that general communication and coordination processes of knowledge work vs. task-focused processes are not well supported by current technologies.

In short we propose a system in which various data is collected from the status of single users and transmitted to other users to an easy-to-use mobile and desktop application. The application supports better group awareness in terms of workspace awareness, group-structural awareness, informal awareness and social awareness.

\section{Group performance and effectiveness}

We hypothesize that our system and applications will influence group performance. However, there is more to groups than performance on task only. For instance Andriessen [7] has divided the key aspects of group interactions as i) performance related to activities and specific tasks aimed at reaching a common goal for the group and ii) group maintenance, i.e. activities aimed at enhancing and building cohesion and trust in the group or to gain status and power in the group.

Regards group awareness discussed above we expect that "better" group awareness (workspace awareness, group-structural awareness, informal awareness and social awareness) will then lead to positive outcomes in group interactions, in both performance and group maintenance.

In addition to group performance another concept, group effectiveness has been proposed to consist of three components: 1. production function: effectiveness is here defined as "the degree to which the productive output meets or exceeds the performance standards set by the clients". Criteria used to define such dimension are product quality, product quantity, efficiency and innovativeness; 2. group well-being function: is defined as "the degree to which the attractiveness and vitality of the group is strengthened"; 3. member support function: "the degree to which participating in the group results in rewards for the individual group members" [6, pp 100].

We propose that different types of group awareness are linked to group performance and group maintenance and are prerequisites for group effectiveness.

Previous studies have shown that there are several possibly central concepts that are related to social interaction in our focus area of group performance. We use the examples of group cohesion, cooperativeness or reciprocity and convergence below.

Group cohesion has referred to as "the extent to which group members perceive and feel attracted to the group" [6, pp 118]. Cohesion in a group has also been described as "a tendency to stick together or to be united either physically or logically" [8, pp 130].

Another important dimension in defining the quality of a social interaction is cooperativeness, a kind of reciprocity of communication. It is described as the behavior of people towards others in the group with which they share common interests and tasks. The behavior is also characterized by the fact that each person strives towards their goals within the group and this progress is facilitated by the other person's actions, leading each to expect reciprocation [9]. 
There is also another interesting approach to seeing how groups communicate: conveyance and convergence. Conveyance can be seen as the exchange of information among participants of the group in which the interpretation of the messages is done by the receiving individual [10]. This refers to the individual act of receiving and interpreting messages. Convergence, on the other hand, is about producing and facilitating shared social meanings among participants, rather than individual interpretations.

In previous studies it has been suggested that these two different dimensions of meaning-making in a group may emerge differently based on the different mediating technologies used. People in face-to-face interactions tend to reach consensus (high level of convergence) faster than groups using online chatrooms [11]. It has been proposed that synchronous media are better in facilitating convergence whereas asynchronous media are better in supporting conveyance [12]. It then seems that it may be sensible to think of solutions to enhancing cohesion, cooperativeness, the efficient emergence of shared meanings and convergence as part of group awareness in the group in the context of technological support for group work. We have tried to address these issues in our prototypes with several ways of creating and sharing group awareness.

\section{Activity indexes for a working group}

For our prototypes we have preliminarily selected a number of key activity indexes that describe the state of the group or its activities. The indexes are formed by analyzing the user's activities through our system. The indexes are displayed to users via our applications in visual representations.

We have preliminarily chosen among many potential indexes which would be useful in group work. It should be noted that we have not yet tested our chosen indexes in field tests within the context of our applications and that the indexes after our first field tests could be altered, rejected or changed.

Our chosen indexes for group activity are: group reciprocity, group centralization, my participation, my reciprocity, my popularity and my influence power. These indexes are hypothesized to be related to different types of group awareness. We expect that making such indexes available in a group work situation therefore enhances certain types of group awareness. In more detail the indexes and their meanings are as follows divided into group and individual levels:

\section{A. Group level indexes:}

\section{Cooperation level}

Description: Describes the collaboration level in the group. Based on the tendency to contact others within the group in work tasks. A group could at certain times be very highly collaborative and at some times very little. This could be used to give an understanding the activity level and "tightness" of the group.

Types of group awareness supported: Workspace awareness: what is the activity level of the group, what is the context into which I am sending my messages or requests? Social awareness: What is the level of "attention" of the group, how intensively the group works together? 


\section{Communication hierarchy}

Description: Describes whether the group activity reflects a tendency to be hierarchical (top-down) around some individuals or whether it is democratic (many-tomany). In other words it is like the "power structure" of the group.

Types of group awareness supported: Group-structural awareness: Who is the intellectual leader of the group? Who provide the best ideas? Is the group really collaborative and democratic or really top-down led by a single individual, perhaps corporate supervisor of the group? What are the different roles of people in the group?

\section{B. Individual indexes:}

\section{My influence power}

Description: Describes how much a person is involved in the actions of the group. Can be based on how many contacts or messages the user receives. This reflects the importance of the user in the group.

Types of group awareness supported: Group-structural awareness: How central am I as a person in the group? How central are others in the group? Do I have a lot of influence over the group? Who has most and least influence over the group?

\section{My popularity}

Description: Describes the user's popularity based on the idea that a user is more popular the more messages he/she receives.

Types of group awareness supported: Group-structural awareness and social awareness: Am I popular? Who is the most popular person in the group? How popular is the person I am sending my message to? How should I craft my negative feedback message to this person as he is so popular in the group?

\section{My participation}

Description: Describes how actively the user participates in the activity of the group. Is assessed on how much activity the user inputs into the system.

Types of group awareness supported: Group-structural awareness: How actively do I participate in the groups activities? How active is my boss or co-worker?Social awareness: What is the level of interest of a person in the task we are doing based on his participation index?

\section{My reciprocity}

Description: Describes how much the user is mutually contacted when the user contacts others. It reflects a kind of symmetry or mutuality of communication as seen from the point of view of a single user. For instance, a user could be communicating a lot but not receiving a lot of feedback, or the user's sent and received messages reflect a balance or communication activities.

Types of group awareness supported: Social awareness: What is the attention level of other users I am contacting regards my messages? Do they reply to my messages actively or not at all? Is there someone I am ignoring? Am I isolated in this group and if so, why? Should I talk more to this person as I have not really communicated with him? 


\section{Prototype System and Application}

\section{Mobile Application}

We constructed two prototypes based on our approach to designing the system: a mobile application running on mobile phones and a desktop application running on top of Microsoft Outlook. These two applications are the first ones constructed and reflect the status of the project at the time they were built. First we will discuss the mobile application.

\section{Technical description: client}

The client is based on Flash and Python technologies. The Flash part of the application is basically for handling vector graphics and showing results that come from the server. Python handles the hardware devices like Bluetooth and camera. We used Py60 for the Symbian platform and PyCE for WM.

The Flash/Python application is able to run in many platforms such as Symbian phones (S60 2nd 3rd editions), WM, desktops (Linux/Windows/Mac OSX) and PocketPC.

\section{Technical description: server}

Server is based on Python/PHP. The main computations for social network analysis (SNA) indexes are done by the PSE, a special software that analyses and generates the social indexes discussed earlier. The interaction with PSE happens through a special protocol - SOAP - and the SOAP client is PHP based.

\section{Interface description}

When starting, the application updates data and this may take some time. The application opens to an orbit view (see Figure 1, column A). In this view the members of the group are placed to a three concentric circles with the user in the middle. Other group members are placed to the orbits around you either by "My Participation" or by "My Reciprocity" indexes. A person on the innermost orbit has similar index values with the user, whereas a person on the outermost orbit has very dissimilar values with the user.

The user is given explanations of all the icons shown in Figure 1. For example MyParticipation is explained as ollows My participation refers to how extensively involved a person is in the communication among the groups members. Similar explanations and icons are used for other indexes.

Left and right buttons in the mobile phone are used for rolling the circles to select a user, for the selected user the user name is highlighted. A firing button press opens a details window for the group member that has been selected. In the details window following data are shown (Figure 1, panel D): user name, availability and time tag, activity: a free text field for the current activity, last used: a time tag for when the user last time launched the mobile application, nearby: a list of nearby group members, based on mobile phone's Bluetooth proximity, also, a user taken photo, with a time tag, is shown.

The details window can be shut by pressing again the firing button. The Actions menu under the right soft key (Figure 1, panel E) offers sending a private message to the selected user, calling to the selected user, or sending him/her a sound message. From the actions menu the application can also be put to the background or closed. 
For some phone models it is not possible to put applications to the background, in these cases the only way is to shut the application, if other phone functionality is needed, and then again restart it.

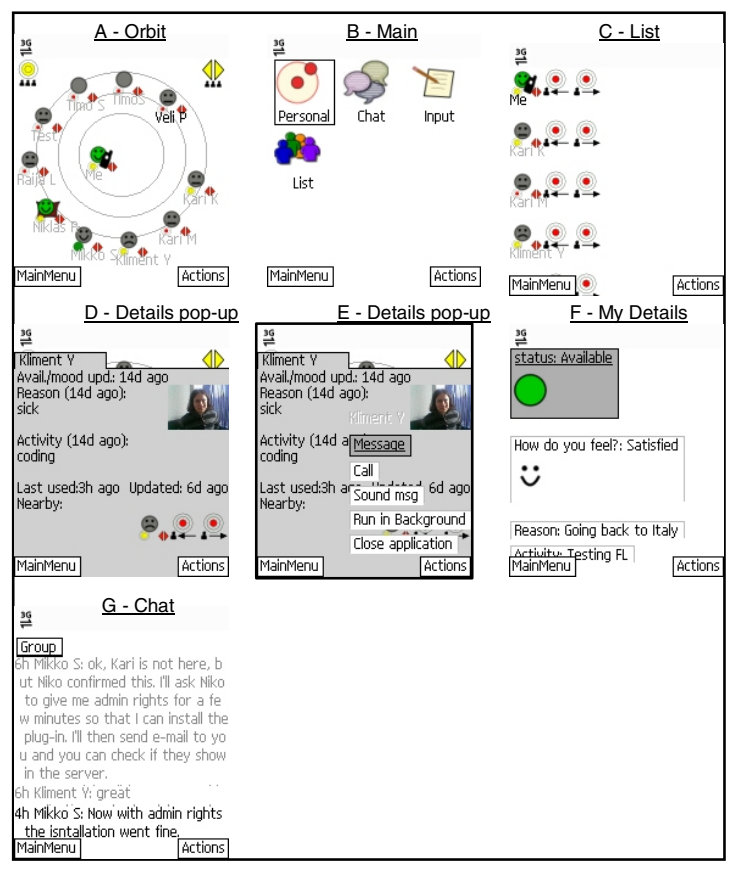

Fig. 1. Different views of the first version of the mobile application. A) The orbit view. B) Main menu. C) List view. D) Details pop-up window for a single user data. E) Details pop-up window with the actions menu opened. F) My details view for inputting availability information etc. G) Chat view.

Pressing the Main with the left soft key opens the Main menu (Figure 1, panel B). Here the user can choose between the previously described orbit view, the List view, Input menu, and the Chat area. In the list view the list of group members is sorted with the same criteria as in the orbit view. Pressing the firing button opens the details pop-up window for a single user.

In the My Details the user can input his/her status, mood, reason, action and launch the camera for taking pictures (Figure 1, panel F). On some phone models it is not possible to take pictures through the application. In the chat area the messages are preceded with a time tag (for example $1 \mathrm{~h}=$ the message was sent 1 hour ago) and a user name (Figure 1, panel G).

\section{Desktop Application}

The desktop application works through an Outlook plug-in with Microsoft Outlook 2003 (and Outlook 2007 to a limited degree) and provides augmentation to the mail client on the basis of emails sent within the application. Additionally, Internet Explorer 7 or Mozilla Firefox 2.x is required for the Outlook tab to work properly. 
After the installation the user should be able to see the Pasion button (i.e. the button for our application to be launched) below the standard command bar (where there are icons to create a new document, open, save etc.) in Outlook. The Outlook plug-in collects data about e-mail traffic between trial group members and sends it to the Pasion server (i.e. our application server). The collected data are sender, recipient, time and date. It should be noted that no information on the content of e-mails is collected

The data collected by tour server is used in calculation of the chosen indexes. These indexes are visualized both in the Outlook plug-in and in mobile application. The indexes are calculated from pooled e-mail and chat messages.

In the upper left panel of the Outlook tab is a list of group members (Figure 2). Pressing Sort list in the upper left panel opens the sorting criteria menu. The user list can be sorted by Username, My Participation (automatically calculated index, described above), My Reciprocity, My Popularity, My Influence Power, Mood (manually set variable), and Availability (manually set variable).

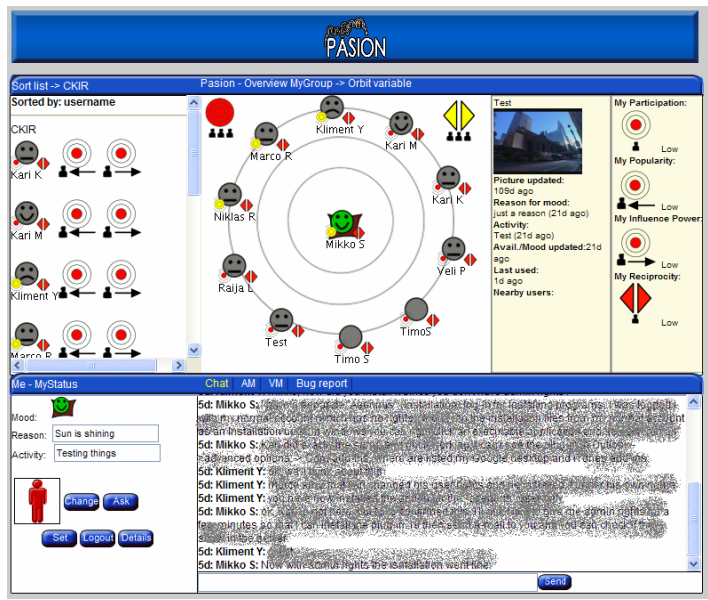

Fig. 2. First version of desktop application interface in Outlook

Placing the mouse pointer over the user icon in the list view opens a pop-up window. From this window it is possible to send the user a private instant message, or view the user's detailed information.

Below the group panel is the MyStatus panel. Where the user can set his/her mood, reason, activity, or download a picture which is then shown in the pop-up details window. It is also possible to ask other group members to update their mood, reason, activity, or picture by placing the cursor over the Ask button. The request to update will be sent to the chat area. After editing the values the Set button must be pressed for the values to update. A logout button is also located there. Pressing the details button opens a view where the user can change password, language (English, Finnish, Italian, and Spanish), phone number, Bluetooth address, and e-mail. 
The orbit view operates similarly as with the mobile application, described above. Bringing a cursor over a user shows the details window on the right. A private instant message can be sent to user by clicking him/her. A separate tab is opened for private messaging to each group member. These can be closed from the chat areas title bar. In the details pop-up window there are shown: the user name, user taken picture, reason for mood, activity, last used time tag and nearby users determined by the Bluetooth proximity. The shown indexes are: My Participation, My Popularity, My Influence Power, and My Reciprocity.

\section{Discussion}

One of the key future challenges for our work is to identify tasks that most benefit from the use of our system. After identifying the tasks we can redesign our system to support them and optimize the use of increased group awareness to fit the purpose. We hope that our initial emphasis on general communication processes across several types of tasks in knowledge work teams will produce results that help to focus our work further.

There may also be challenges in the area of privacy when using our system. For instance, we hypothesize that no single user would wish to publicly transmit their social information.

Similarly, problems of social comparison may arise. This has at least two sides. First, if a user is always in the periphery of the group as indexed by our visualizations in different knowledge work tasks, it may indicate unpopularity, not being liked, or not being valued. Second, as this is a system for work, there is always the question of one's value to the employer. If the social network analysis visualizations show to one's boss that one is constantly not producing much input and is at the periphery of interactions at a task in which one should perhaps not be at the periphery, this may create doubts about the role and value of such a person to the employer.

On the other hand, both problems described above regards social comparison also cut the other way. For instance, if someone is very central and active as indexed by our system in some knowledge work task, it informs the person as well as the boss of his popularity and value to employer. In this way one could perhaps have "hardcore data" of one's performance within the group and in the eyes of the employer. At the group level, it could be hypothesized that people tend to pump-up their performance relative to others and this would have positive outcomes regards group performance in total.

However, the main problem may be the social mirror- effect. The indexes used create an individual mirror image in terms of: "Who am I in the context of this group? What is my worth? Am I popular and liked? Am I effective at my tasks?" Our system can also create an image of the others in the system, such as: "Who is this popular person? Why is this person the center of our communication? Why is this person at the periphery of our discussions when I expected more?"

Our system creates a mirror image on some dimensions of the group using the system. This image can be either flattering or not. Despite the obvious challenges we feel that users will benefit from the use of our system by gaining a more holistic view of the group they are working with in addition to gaining insights about themselves in 
relation to the group. Our rationale is to enable users to transmit and receive enriched social cues to enhance their communication processes while working. The intrusiveness, resolution and accuracy of gathering the information as well as the understandability of visual representations of various indexes are naturally critical issues.

The next stage of our work is to run field tests of our application in real-life working environments. Based on this research a new, refined version of the system will be built with tested and selected functionalities and improved visualization schemes.

\section{References}

1. Senge, P.: Sharing knowledge. Executive Excellence 14(11), 17-18 (1997)

2. Davenport, T., Jarvenpaa, S., Beers, M.: Improving knowledge work processes. Sloan Management Review 37(4), 53-65 (1996)

3. McGrath, J.E., Hollingshead, A.B.: Putting the "group" back in group support systems: Some theoretical issues about dynamic processes in groups with technological enhancements. In: Jessup, L.M., Valacich, J.S. (eds.) Group Support Systems: New Perspectives, pp. 78-96. Macmillan, New York (1993)

4. Greenberg, S., Gutwin, C., Cockburn, A.: A using distortion-oriented displays to support workspace awareness. Technical report, Dept. of Comp. Science, Univ. of Galgary, Canada (January 1996)

5. Saari, T., Kallinen, K., Salminen, M., Ravaja, N.: A System for Facilitating Emotional Awareness in Mobile Knowledge Work Teams. In: 41st Hawaii International International Conference on Systems Science (HICSS-41 2008), Proceedings, Waikoloa, Big Island, HI, USA, January 7-10, 2008. IEEE Computer Society, Los Alamitos (2008)

6. Woodman, R.W., Sawyer, J.E., Griffin, R.W.: Towards a theory of organizational creativity. Academy of Management Review 18(1), 293-321 (1993)

7. Andriessen, J.H.E.: Working with groupware. Understanding and Evaluating Collaboration Technology. Springer, London (2003)

8. Reber, A.S.: The penguin dictionary of psychology. Penguin, London (1985)

9. Raven, B.H., Rubin, J.Z.: Social psychology: People in groups. Wiley, New York (1976)

10. Wheeler, B.C., Dennis, A.R., Press, L.I.: Groupware comes to the Internet: charting a new world. ACM Sigmis Database 30(3-4), 8-21 (1999)

11. Dennis, A.R., Valacich, J.S.: Beyond media richness: an empirical test of media synchronicity theory. In: Proceedings of the Thirty-Second Hawaii International Conference on System Sciences, vol. 1 (1999)

12. Hung, Y.T.C., Kong, W.C., Chua, A.L., Hull, C.E.: Reexamining media capacity theories using workplace instant messaging. In: Proceedings of the 39th Annual Hawaiii International Conference on System Sciences, vol. 1, 19.2 (2006) 